Article

\title{
Self-Fitting Algorithm for Digital Hearing Aid Based on Interactive Evolutionary Computation and Expert System
}

\author{
Ruiyu Liang ${ }^{1,2, *}$, Ruxue Guo ${ }^{1}$, Ji Xi ${ }^{1,3}$, Yue Xie ${ }^{1}$ and Li Zhao ${ }^{1}$ \\ 1 School of Information Science and Engineering, Southeast University, Nanjing 210096, China; \\ grx0904@sina.com (R.G.); xijie952611@gmail.com (J.X.); hhucxieyue@sina.com (Y.X.); \\ zhaoli@seu.edu.com (L.Z.) \\ 2 School of Communication Engineering, Nanjing Institute of Technology, Nanjing 211167, China \\ 3 School of Computer Information and Engineering, Changzhou Institute of Technology, \\ Changzhou 213002, China \\ * Correspondence: 1ly1711@163.com; Tel.: +86-153-8092-0360
}

Academic Editor: Lorenzo J. Tardón

Received: 13 January 2017; Accepted: 7 March 2017; Published: 9 March 2017

\begin{abstract}
The traditional hearing aid fitting method, which mainly relies on the audiologist, is time-consuming and messy. To improve this situation, a self-fitting algorithm based on an improved interactive evolutionary computation (IEC) algorithm and expert system, which enables the patients to fit the hearing aid by themselves, is proposed. The algorithm takes the band gain as the fitting target and uses the patient's subjective evaluation to iteratively update the algorithm parameters based on the improved IEC algorithm. In addition, a real-time updated expert system is constructed to assist in the optimization of the initial and iterative parameters of the fitting based on the patient's audiogram and personal information. To verify the performance of the algorithm, a self-fitting software for the hearing aid is designed. Through this software, the test signal is generated for the patient to evaluate the audio quality on a five-level scale. Based on the evaluation results, the algorithm iteratively optimizes the algorithm parameters until the patient is satisfied with the generated audio. Compared with the fitting algorithm based on Gaussian processes algorithm or the interactive evolutionary algorithm, the average subjective speech recognition rate of the proposed algorithm increase at least $11 \%$. The average recognition rate for environmental sound is also improved by at least $2.9 \%$. In addition, the fitting time of the proposed algorithm is shortened by at least $10 \mathrm{~min}$ compared to others two algorithms.
\end{abstract}

Keywords: hearing aid; self-fitting; interactive evolutionary computation; expert system

\section{Introduction}

Hearing impairment is the third most frequent chronic disease [1]. Furthermore, hearing rehabilitation is expected to face more severe challenges due to the ageing of the global population $[2,3]$. Currently, the use of hearing aids is one of the most effective means of hearing rehabilitation [4]. However, even in developed countries, only one in five people has hearing aids [5]. Due to more limited medical conditions and lower health-care consciousness, this proportion is much lower in developing countries [6]. One factor that affects the penetration rate of hearing aids is a lack of hearing professionals $[7,8]$, whose skills directly affect the hearing aid fitting process and after-sale service quality.

To this end, teleaudiology is a solution to the problem of the shortage of hearing professionals [5]. This technology is primarily applied in remote hearing diagnosis, hearing testing, regulation and 
consultation for hearing aids and can effectively overcome certain shortcomings, such as long distance, high cost, and lack of professional institutions in remote areas and thus increase access to hearing services [9]. Surveys show that $75 \%$ of hearing-impaired patients with moderate to extremely severe hearing loss are more willing to use remote hearing services [10]. Unfortunately, because doctors and patients represent different countries, many urgent problems remain to be solved, such as qualification, responsibility, reimbursement, and quality control, among others.

From design conception, self-fitting is the best choice for solving the problem of a shortage of hearing professionals [11]. The essence of self-fitting is that the installation, fitting and use of hearing aids are completed by the user alone, without any hearing experts or equipment [12]. In 1984, Köpke, Wiener, et al. proposed this concept [13], but it has not yet been fully achieved. Certain similar products called user-programmable hearing aids have been introduced. However, the design of user-programmable hearing aids is relatively simple. The user manually adjusts the hearing aid using the configuration switch according to his/her subjective judgment of the environment. Studies show that in different environments, the patient can control four parameters $[14,15]$ to realize the optimal configuration or use four independent memories to adjust the algorithm gain, noise reduction mode, microphone mode and spectral enhancement extent [16]. The design concept of the user-programmable hearing aid is similar to that of the self-fitting hearing aid, i.e., the user manually adjusts the parameters of the hearing aid to compensate for the deterioration of speech with changes in the environment. The difference between these two categories of hearing aids is that the number and extent of change of the adjustable parameters for the user-programmable hearing aid are limited, whereas those of the self-fitting hearing aid are not.

Currently, in the commercialization of self-fitting hearing aids, the design of professional instruction materials [17], research on ear moulds, batteries and distribution models, among other activities, are performed simultaneously. With respect to the self-fitting algorithm, parameter optimization of the loudness compensation algorithm was initially realized by Takagi in 2007 based on an interactive evolutionary computation (IEC) [18] algorithm. This study delivered a feasible approach to realizing the hearing aid self-fitting algorithm. Takagi et al. designed a two-dimensional Gaussian distribution optimization model and set the model parameter as the chromosome. Once the model parameters are determined by the IEC algorithm, the fitting model is determined. Because this algorithm has a large solution population and searches for the optimal solution in the global three-dimensional space, the convergence time is inevitably long. Therefore, it is difficult to meet the needs of patients in practical applications. In 2015, a self-fitting algorithm based on Gaussian Processes (GP) and active learning was proposed [19]. The user chooses which of two particular hearing aid settings that is preferred based on active learning algorithm, which can reduce the required number of training examples by sequentially. However, the algorithm is not optimal because it is designed without considering the individual and historical data.

To improve the abovementioned problem, an improved self-fitting algorithm is proposed. The algorithm takes the gains of different frequency bands as the chromosome parameters of the IEC algorithm. Because the high randomness of the parameter optimization process leads to user fatigue, an expert system is constructed to assist in the parameter decision. The main functions include the following: (1) In the early fitting stage, the initial parameter is determined based on the patient information; (2) During the evolutionary process, the individual parameters that most closely match the user information are selected from the knowledge base of the expert system and replace the worst individual parameters. At the same time, based on the analysis of the evolutionary population, parameters such as crossover and mutation probability are modified in each iteration. To verify the algorithm effect, a self-fitting hearing software is designed, and the corresponding subjective listening tests for hearing-impaired patients are completed.

This paper is organized as follows. Firstly, the design concept of self-fitting is introduced in Section 2. Subsequently, the design and implementation of the algorithm are detailed explained in 
Section 3, and the experimental results are shown and discussed in Section 4. Further discussion and prospects for the future are discussed in Section 5. Finally, the conclusions are presented in Section 6.

\section{Design Concept of Self-Fitting}

Because the hearing ability, cognitive level and personal habit of each patient are different, each hearing aid is individually fitted and unique. Although the fitting of a hearing aid is similar to that of glasses, the process is more complex and the practitioner requires higher professional skills. Figure 1 shows a comparison of the fitting processes between a traditional hearing aid and a self-fitting hearing aid. From the figure, during the traditional fitting, the audiologist is highly important and should have rich professional knowledge of hearing aids and audiology. Firstly, the audiologist performs an overall assessment for the patient and selects the appropriate equipment and scheme for aural rehabilitation. Secondly, the audiologist receives feedback from the patient and subsequently determines which parameters need to be adjusted. Finally, the audiologist decides on the extent of the adjustment according to experience. Therefore, in a traditional fitting, the audiologist's skills and experiences are the key to the performance of the hearing aid, which is also one of the important factors affecting the popularity of hearing aids in developing countries.

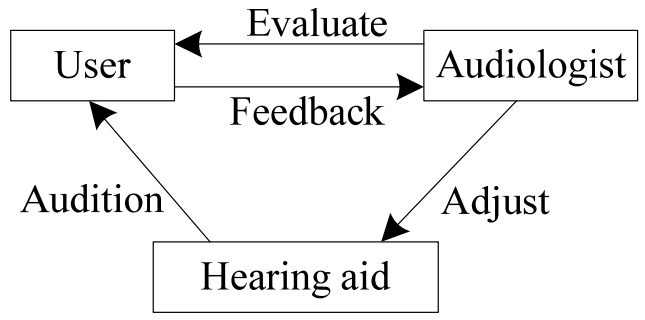

(a)

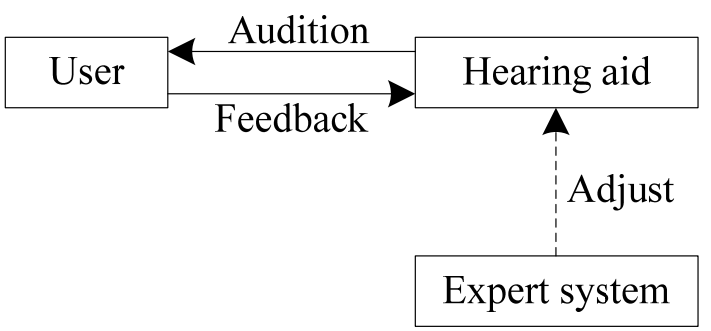

(b)

Figure 1. Different fitting processes for hearing aids. (a) Traditional fitting process; (b) Self-fitting process.

As shown in Figure 1b, during the fitting process, the self-fitting hearing aid can interact directly with hearing-impaired persons without the participation of audiologists. The optional expert system can partially act as an expert based on the artificial intelligence algorithm. The realized functions include parameter initialization, parameter update and hearing measurement, etc. However, related research is relatively scarce. In addition, for portability considerations, the human-computer interaction tends to be wireless, which requires greater power consumption.

Most initial hearing aid parameters are calculated according to the prescription formula, and the goal is to obtain the average configuration of the hearing aid according to certain specific parameters [20,21]. However, individual differences such as age, sex or service life of the hearing aid affect the fitting results, and thus the average approach is biased [22]. Therefore, how to effectively adjust the parameters is quite difficult $[23,24]$. Because the basic algorithm for the hearing aid is the multichannel loudness compensation algorithm [25], its parameter fitting is discussed in the following subsections.

\section{Design and Implementation of the Algorithm}

\subsection{Overall Structure}

The self-fitting algorithm is a process of interaction between subjectivity and objectivity. In addition to ensuring the performance of parameter optimization, reducing user fatigue is also highly important. To improve the efficiency of parameter optimization, two improved strategies are proposed: one strategy builds an expert system to initialize and optimize the parameters of the algorithm and the other improves the genetic algorithm to increase the efficiency of the iterations. 
The overall self-fitting process for hearing aids is shown in Figure 2. The system includes three components: hearing aid test system, patient and self-fitting system. The basic workflow is as follows. Firstly, the input sound is processed by the algorithm in the hearing aid test system, and the processed sound is presented to the user. Secondly, the user evaluates the sound quality according to subjective criteria. Finally, the evaluation results are fed into the self-fitting system for parameter optimization and adjustment, and the new parameters are returned to the hearing aid test system to generate the new sound. As seen in the figure, the self-fitting system is the key, which is composed of three components: user interface, evolution module and expert system.

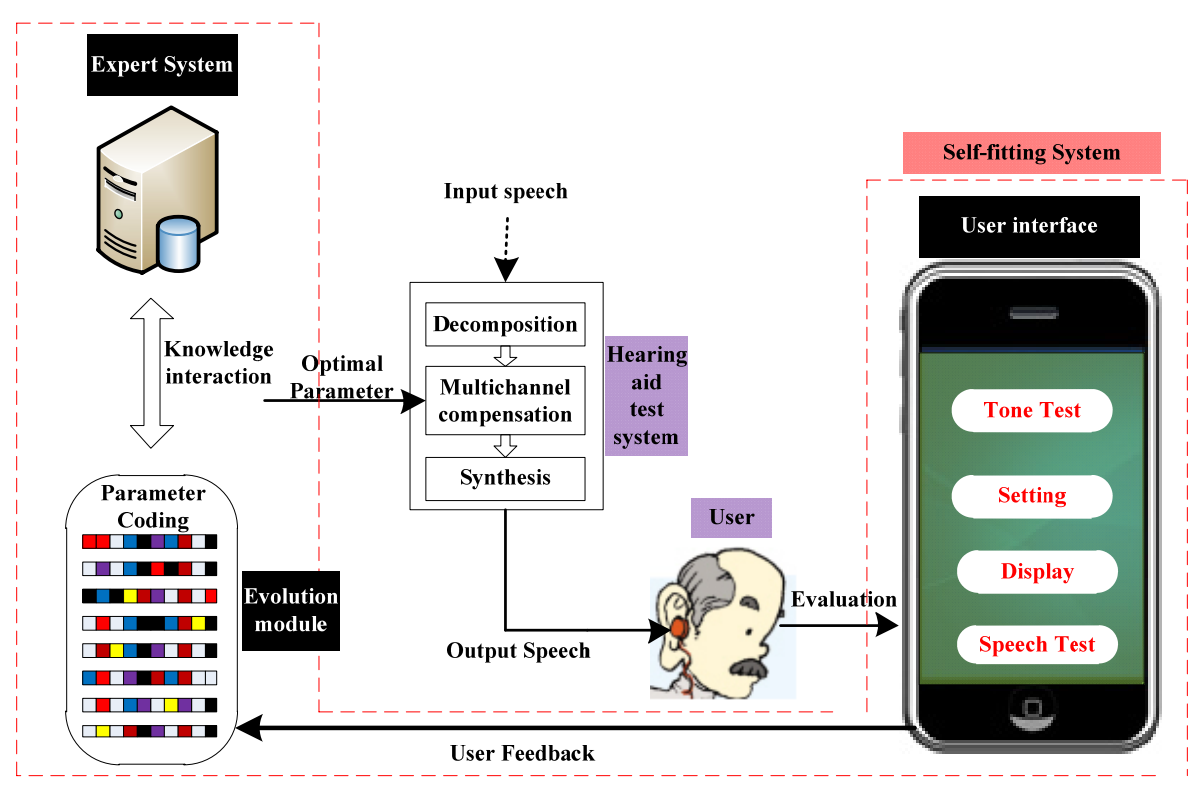

Figure 2. Overall structure diagram of self-fitting.

The user interface is the only conduit for human-computer communication between the user and the self-fitting system. Its main functions include hearing threshold measure for patients and sound quality evaluation. The hearing threshold measure is implemented based on simplified pure tone audiometry. The basic test procedure is the same as the traditional tone test [26], and the measured tones are 125, 250, 500, 750, 1000, 1500, 2000, 3000, 4000, 6000 and $8000 \mathrm{~Hz}$. After the tone frequencies are tested, an audiogram of the hearing-impaired person is obtained. The sound quality evaluation is used to assess the generated sound and feed the assessment results back to the evolution module. The assessment results for the sound quality have five levels: bad, poor, medium, good and excellent.

The evolution module is the core of the self-fitting system. Based on the user feedback on the sound quality, the module updates the algorithm parameters based on the proposed evolution algorithm. In this work, the updated parameters are the sub-band gains. Based on these parameters, the evolution module generates the new speech signals and presents them to the user to reassess.

The expert system analyses the user preferences and calculates the diversity of the population, which forms the corresponding knowledge and feeds it back to the evolution module. Firstly, according to the audiogram and the personal attributes (age, sex and duration of hearing impairment) of the patient, the module initializes or constrains the algorithm parameters to improve algorithm efficiency, and secondly, it interacts with the evolution module to accelerate convergence.

\subsection{Evolution Module}

The evolution module is designed based on the improved IEC algorithm. Because the self-fitting algorithm requires high efficiency to reduce user fatigue, the high randomness of parameter mutation in 
the traditional genetic algorithm is unreliable. Therefore, the parameter iteration process is optimized to accelerate convergence according to different patients. The algorithm flowchart is shown in Figure 3.

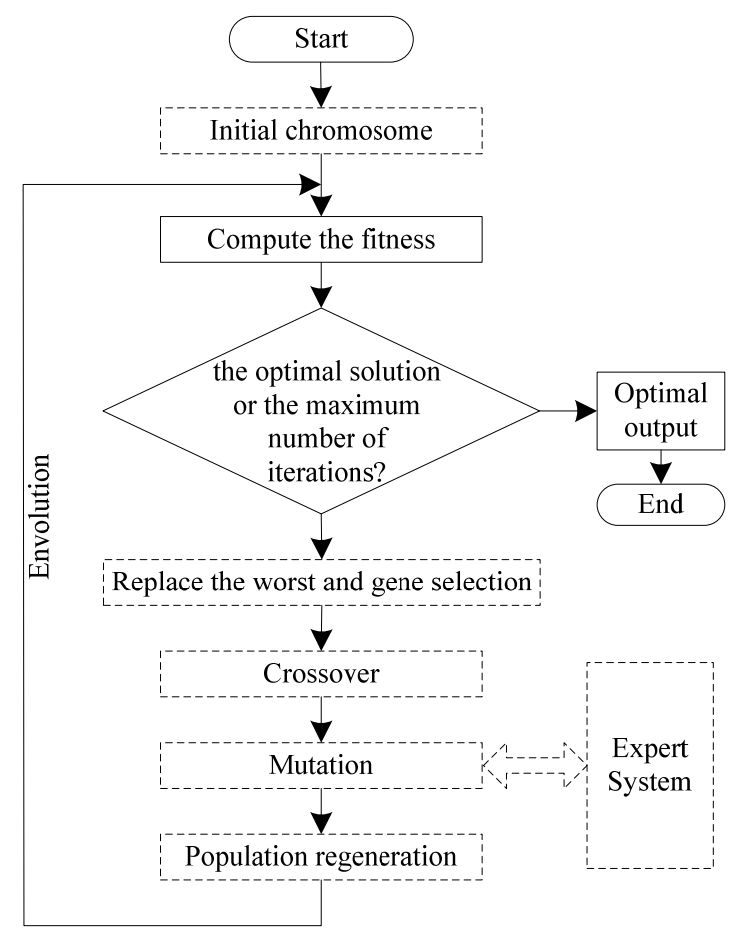

Figure 3. Flowchart of the improved genetic algorithm.

(1) Initialize the chromosome population. The algorithm parameter is encoded in binary, which is defined as the structural data of the genotype (i.e., chromosome). According to the patient's age, sex, duration of hearing impairment, and audiogram, $M-1$ historical patients whose information is closest to that of the current patient's information (minimum Euclidean distance) are selected from the expert system. Here, $M$ is the population size. The parameters of the selected patients and the last group of parameter based on the prescription formula NAL-NL2 [22] construct the whole $M$ parameters.

Even with the same audiogram, the real fitting results are influenced by the patient's cognitive level and personal habits, among other factors [22]. So, the proposed self-fitting algorithm optimally sets the signal gain set according to the patient information [25] and feedback. The signal gain in every frequency band is taken as a gene. Because the gain ranges from 0 to $120 \mathrm{~dB}$, it is coded in 7-bit binary.

(2) Compute the fitness of the chromosome. For the self-fitting algorithm of the hearing aid, fitness is determined based on the feedback from the patient. Considering the evaluation resolution and user fatigue, the fitness is divided into five levels: bad, poor, medium, good and excellent. Every level is represented in the specific value. The evaluation scale is highly important to the practicality and convergence of the interactive fitting. If the evaluation scale is too accurate, the burden of the algorithm is too large to cause user fatigue. Instead, although it is easier for the user to accept a rough evaluation, a larger quantization error leads to low convergence. Earlier research showed that the five-level evaluation scale is a compromise selection [27]. The performance of the algorithm is directly affected by the numerical selection, and thus the fitness values are selected based on the hyper-geometric operator. The genetic probability of each level is described as:

$$
P_{n}=\frac{1}{\gamma \times n^{q}}
$$


where $\gamma$ is set as $\sum_{k=1}^{5}\left(1 / k^{q}\right)$, and $q$ is a constant of 0.5 . Use of the genetic probability as the fitness can make the genetic algorithm more stable. In addition, the prematurity problem can be avoided to a certain extent.

(3) Calculate the optimal solution. If the current chromosome is determined as the optimal solution or the maximum number of iterations is reached, the process ends. Otherwise, the optimization proceeds. When the iterations end, the output is the optimal solution in the iteration process.

(4) Gene selection. The individuals with higher fitness in the current population are transmitted to the next-generation population according to a certain rule. In this work, the roulette selection rule is applied, i.e., the probability that the individual is selected and transmitted to the next generation is proportional to the individual fitness. One circle is divided into $M$ pieces according to the probability $P_{n}$. During selection, the circle is rotated, and if a point falls into the $i$-th sector, then individual $i$ is selected. The characteristic of the roulette selection rule is that each individual has an opportunity to be selected.

(5) Genetic crossover. Selected chromosomes from two individuals are exchanged with probability $P_{c}$ to obtain two new individuals.

The algorithm uses the single-point crossover function. Chromosome pairing and the crossing point selection are two key factors. The function of chromosome pairing is to avoid the crossover of two similar chromosomes, which affects the search for the optimal solution. In addition, different crossing points lead to different effects for the same crossover pairs. In the worst case, if the new chromosome pairs after crossover are the same as the original pairs, the operation is invalid. To avoid this problem, an improved method is adopted.

(a) Before the chromosome is paired, the correlation between two chromosomes is firstly analysed. The uncorrelated exponent of chromosome $x_{l}=\left\{g_{l 1}, g_{l 2} \ldots g_{l N}\right\}$ and $x_{m}=\left\{g_{m 1}, g_{m 2} \ldots g_{m N}\right\}$ is defined as:

$$
r\left(x_{l}, x_{m}\right)=\sum_{k=1}^{N} g_{l k} \oplus g_{m k}
$$

The selection process for chromosome pairing is as follows. First, the chromosome pairing pool (the pairing pool includes all chromosomes that have not been paired in a population) is defined as $\left\{y_{1}, y_{2}, \ldots, y_{S}\right\}$, and $S$ is the number of chromosomes. After one chromosome $x$ is randomly selected, another chromosome $y_{i}$ is selected from the pairing pool to perform the crossover operation. If the difference between the selected individual and the others in the pool is not clear, the efficiency of the crossover operation is greatly affected. Therefore, the unequal probability pairing strategy $[28,29]$ is used. The individual with the larger uncorrelated exponent in the pairing pool is set with the greater probability, and the probability $P\left(y_{i} / x\right)$ with which the individual $y_{i}$ is selected to pair with the individual $x$ is defined as follows:

$$
P\left(y_{i} / x\right)=\frac{1}{S}\left(1+\lambda \frac{r\left(x, y_{i}\right)-r_{a v g}}{r_{\max }-r_{\min }}\right), i=1,2, \ldots, S
$$

where $\lambda \in[0,1]$ is a constant of $0.8, r_{\text {avg }}=\frac{1}{S} \sum_{i=1}^{S} r\left(x, y_{i}\right), r_{\max }$ and $r_{\min }$ are the average, maximum and minimum values of $r\left(x, y_{i}\right)$, respectively. If $r\left(x, y_{i}\right)>r_{a v g}$, the probability of selecting $y_{i}$ is larger than the average probability. Otherwise, $y_{i}$ is smaller than the average.

(b) The selection of the crossing point is as follows. Firstly, the effective intersection area is determined. Secondly, one position is randomly selected as the crossing point in the effective intersection area of $\left(n_{\min }, n_{\max }\right)$, which is determined by the following formula: 


$$
\left\{\begin{array}{c}
n_{\min }=\min \left\{k / g_{l k} \neq g_{m k}, k=1,2, \ldots, N\right\} \\
n_{\max }=\max \left\{k / g_{l k} \neq g_{m k}, k=1,2, \ldots, N\right\}
\end{array}\right.
$$

In the crossover operation, if a coarse granularity and large step search strategy is adopted, it is favourable for global searching but has a negative effect on the local search performance. In addition, the role of the mutation operation is to compensate for the adverse effects of the crossover operation and to maintain the diversity of population. However, the performance of the fixed mutation probability used in the traditional genetic algorithm is poor in the later stages of the algorithm. Based on the above reasons, an adaptive crossover mutation algorithm is used. If each individual fitness value tends to be consistent or similar to the local optimal solution, the crossover and mutation probability is increased to pick out the local optimal solution. If the group fitness of the population is dispersed, the crossover and mutation probability is reduced to retain the excellent chromosomes. The self-fitting crossover formula is described as follows:

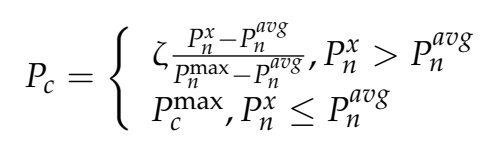

where $\zeta$ is a constant of $e^{-0.382}, P_{c}^{\max }$ is the upper limit of crossover probability, $P_{n}^{x}$ is the larger fitness value of the two individual chromosomes to be crossed, $P_{n}^{\max }$ is the maximum fitness value of the population and $P_{n}^{a v g}$ is the average fitness value.

(6) Mutation operation. The genes in each individual mutate with probability $P_{m}$, and the probability formula is given as follows:

$$
P_{m}=\left\{\begin{array}{l}
(1-\zeta) \frac{P_{n}^{x}-P_{n}^{a v g}}{P_{n}^{\max }-P_{n}^{a v g}}, P_{n}^{x}>P_{n}^{a v g} \\
P_{m}^{\max }, P_{n}^{x} \leq P_{n}^{a v g}
\end{array}\right.
$$

where $P_{m}^{\max }$ is the upper limit of the mutation probability.

Considering the characteristics of the hearing aid, the mutation steps should be directly related to the types of hearing loss and accelerate the evolution in a beneficial direction for the patient. Because the original shape of the chromosome might be destroyed by the previous crossover operation, serious deviation from the original shape is bound to affect the gain compensation effect for a patient with a particular type of hearing loss. Therefore, the mutated gain of every frequency point does not deviate from the initial optimization value of $20 \mathrm{~dB}$. Otherwise, the gain variation of this frequency point is set as $20 \mathrm{~dB}$. Such a setting complies with the rule of hearing aid fitting, which is to avoid obviously invalid parameters.

(7) Population evolution. After the operations of selection, crossover, and mutation, one new population is obtained. When the population evolves a certain number, the optimization process is terminated.

\subsection{Expert System}

The structure diagram of the expert system is shown in Figure 4. Except that the expert system can improve the initial value according to the patient's information, it also facilitates user preference matching and analysis of population diversity. Based on the existing knowledge base, user preference matching can analyse the user preference and find the chromosome that is most similar to the current user preference [30]. The selected chromosome replaces the one with the worst fitness in the existing population. The chromosomes in the knowledge base are the final chromosomes for the fitted patients. Population diversity analysis is used to prevent the prematurity phenomenon. If the population 
diversity is low, the algorithm increases the mutation probability, the crossover probability and the search step to enrich the current population.

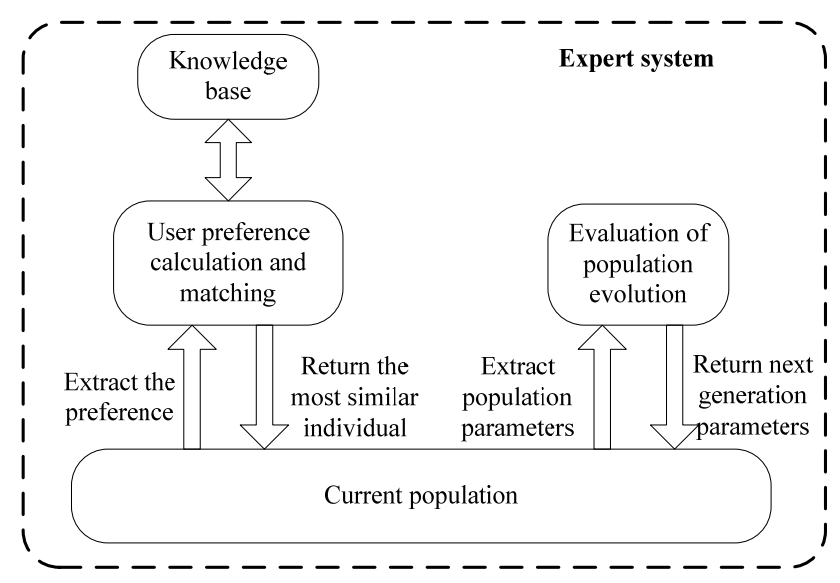

Figure 4. Structure diagram of the hearing aid expert system.

(1) User preference matching

In the IEC algorithm, the user preference for the chromosome is reflected in the individual evaluation. During the self-fitting process, the chromosome that is most similar to the current user preference is selected according to user feedback and is added to the existing evolution population. The chromosome with the worst fitness in the current evolution population is also removed to accelerate the evolution and improve the optimization process.

The chromosome is composed of the gains of different frequencies. For the $i$-th chromosome $x_{i}=\left\{g_{i 1}, g_{i 2} \ldots g_{i N}\right\}, N$ is the number of parameters, and $g_{i k}$ is the $k$-th gain of the $i$-th chromosome, which is expressed in 7-bit binary, and $g_{i k}^{j}$ is the $j$-th bit of the $k$-th gain in the $i$-th chromosome with a value of 0 or 1 . The validity $F\left(g_{k}^{j}\right)$ of the allele unit $g_{k}^{j}$ is described as:

$$
F\left(g_{k}^{j}\right)=\frac{\sum_{i=1}^{N} g_{i k}^{j} P_{n}^{x_{i}}}{\sum_{i=1}^{N}\left(1-g_{i k}^{j}\right) P_{n}^{x_{i}}}
$$

where the evaluation value of the current chromosome $x_{i}$ is $P_{n}^{x_{i}}$, which is shown in Formula (1). Because the fitness evaluation of the chromosome is subjective and the human evaluation might change over time, it is inevitable that the individual evaluation criteria will change during the evaluation process. At the beginning of the fitting, the cognition of the chromosome quality is fuzzy. As the evaluation process proceeds, the patient's psychological standard of chromosome evaluation is gradually unified, and at this time, the evaluation is credible. Therefore, the confidence function $R(N)$ is introduced to simulate the credibility of the subjective evaluation.

$$
R(N)=\left\{\begin{array}{l}
1+e^{-a \times N_{s}}-e^{-a \times N}, N<N_{S} \\
1, N \geq N_{s}
\end{array}\right.
$$

where $a$ is the confidence coefficient. During the process of self-fitting, if the evaluation of speech quality is slow, $a$ should be larger and $N_{S}$ should be smaller. Otherwise, $a$ is larger and $N_{S}$ is smaller. Here, $N_{s}$ is 5 and $a$ is 0.15 . The preference $P\left(g_{k}^{j}\right)$ of the allele unit $g_{k}^{j}$ is adjusted by the confidence function, which is described as follows: 


$$
P\left(g_{k}^{j}\right)=R(N) \times F\left(g_{k}^{j}\right)
$$

The preference similarity $\sigma_{k}\left(u_{1}, u_{2}\right)$ of gene unit $g_{k}$ between the patient $u_{1}$ and $u_{2}$ in the knowledge base is defined as

$$
\sigma_{k}\left(u_{1}, u_{2}\right)=\sqrt{\frac{1}{L} \sum_{j=0}^{L-1}\left(\left(P_{u 1}\left(g_{k}^{j}\right)-P_{u 2}\left(g_{k}^{j}\right)\right) \times 2^{j}\right)^{2}}
$$

Here, $L$ is the bit number of every parameter. Because the gene unit represents the frequency gain in binary form, the weight of different allele unit $g_{k}^{j}$ is not the same. Therefore, the user preference for each bit is multiplied by the factor $2^{j}$. The similarity $\sigma\left(u_{1}, u_{2}\right)$ of the user $u_{1}$ and the matched user $u_{2}$ is defined as:

$$
\sigma\left(u_{1}, u_{2}\right)=\sum_{k=1}^{N} \sigma_{k}\left(u_{1}, u_{2}\right)
$$

The smaller the $\sigma\left(u_{1}, u_{2}\right)$ is, the greater the similarity of the user $u_{1}$ and the matched user $u_{2}$ will be. During the process of population evolution, the user with preferences most similar to the current user preferences is searched in the knowledge base at each evolution. The final fitting parameters of the matched user are added to the current evolution population to replace the worst individual, which hastens the population evolution and decreases user fatigue.

(2) Evaluation of the population evolution

During the process of population evolution, the degree of evolution of the current population is continuously evaluated. The diversity of the population has a great influence on the algorithm performance, which is an important measure of the evolutionary state of the genetic algorithm. If an extremum exists, the same or similar individuals will be produced. It worsens the diversity of the population, which influences the efficiency of the genetic operation and the ability to explore other extreme regions [31]. The population diversity $d$ is defined as follows:

$$
d=1-\frac{1}{0.5 \times N \times L} \sum_{k=1}^{N} \sum_{j=1}^{L}\left|q_{k}^{j}-0.5\right|
$$

where $q_{k}^{j}=\frac{1}{M} \sum_{i=1}^{M} g_{i k}^{j}$ represents the probability that the $i$-th gene in the $k$-th channel is 1 . Here, the range of $d$ is $0 \leq d \leq 1$. The larger the value of $d$ is, the better the diversity of the population will be. If the diversity of $d$ is larger than the threshold $D$ with a value of 0.5 , the crossover probability increases by $5 \%$ based on Formula (5) with an upper limit of 0.95 . The mutation probability increases by $0.5 \%$ based on Formula (6) with an upper limit of 0.3. Otherwise, the crossover probability and mutation probability are obtained according to Formulas (5) and (6).

After many tests, some parameters are obtained as shown in Table 1.

Table 1. Settings for the algorithm parameters.

\begin{tabular}{cc}
\hline Maximum crossover probability & 0.95 \\
Maximum mutation probability & 0.3 \\
Population size $M$ & 8 \\
Maximum genetic generation & 20 \\
Fitness function & Five levels, hyper-geometric operator \\
Diversity threshold $D$ & 0.5 \\
\hline
\end{tabular}




\section{Experiment and Discussion}

\subsection{Experimental Settings}

Speech and other audio data are involved in the experiment. One of the most important features of self-fitting is that any sound could be used in fitting [18,19], unlike the traditional method that uses speech, pure tone or noise. Therefore, some environmental data are used in the fitting evaluation, which quantitatively demonstrates improvement in audio quality. Four types of environmental sounds are used, which include 5 natural climate sounds, 33 daily life sounds, 19 musical instrument sounds and 5 animal sounds. A total of 4-5 similar audio segments are used in every sound, and every sound lasts $5-10 \mathrm{~s}$.

The speech signals are all from the professional listening and fitting software OTOsuite and the TIMIT speech database. The OTOsuite software includes English samples and Chinese samples. English samples include the AB (Arthur Boothroy) word list, BKB (Bamford-Kowal-Bench) sentences [32] and IHR (International Health Regulations) sentences [33], and the Chinese samples [34] include monosyllable word lists, two-syllable word lists and sentences. Because all subjects are Chinese, the Chinese two-syllable word lists are adopted in the experiment.

Seven hearing-impaired patients are tested, 2 females and 5 males, with an average age of 52.4 years (standard deviation 16). The Danish international audiometer AD226 is used to test the audiograms of seven subjects, which are shown in Figure 5. Among these subjects, five subjects have moderately severe hearing loss and two have severe hearing loss.

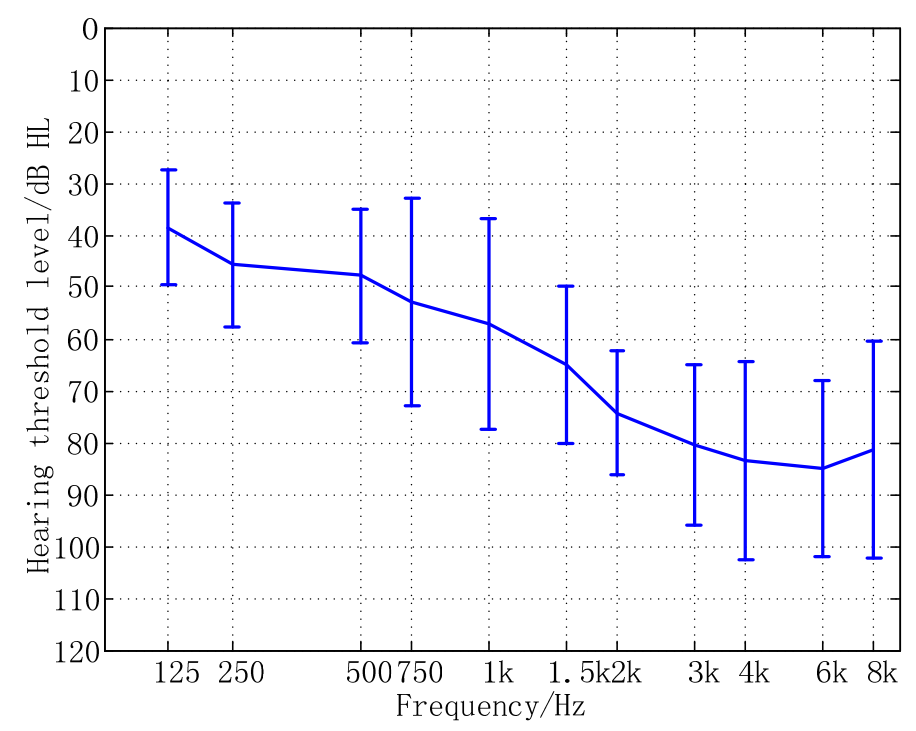

Figure 5. Mean hearing thresholds and SDs for hearing-impaired subjects.

The comparison methods include the proposed fitting method (P), the GP-based algorithm (G) [19] and the IEC-based algorithm (I) [18]. A baseline setting is based on the prescription formula NAL-NL2 [22], which is the initial setting based on the patient's audiogram through the professional fitting software [35]. It is the initial setting of the hearing aids without additional personalization.

\subsection{User Interface}

To verify the performance of the algorithm, self-fitting software is designed based on the Visual Studio 2010 development platform with the main functions of pure tone test and speech test. The pure tone test module can determine the hearing threshold parameter of 11 frequencies ranging from $125 \mathrm{~Hz}$ to $8000 \mathrm{~Hz}$. The speech test module is the main component of the human-computer 
interaction. The pure tone test is performed first, and the test results are used to generate the initial parameters in the speech test.

The interface for the pure tone test is shown in Figure 6. If the test frequency is selected using the drop-down option, the corresponding pure tone is played. If the subject does not hear the sound, the sound intensity button with $\mathrm{dB}$ value is pressed to increase the sound intensity until the sound is heard. The 'Next' button is pressed, and the sound with the new frequency is played. After testing with the 11 frequencies, the hearing threshold parameters for the subject are obtained and displayed in the rightmost side. After the pure tones test, the 'Speech test' button is pressed to enter the interface of the speech test, which is the key human-computer interaction. The 'Exit' button is pressed to end the test if the subject prefers to stop at any point.

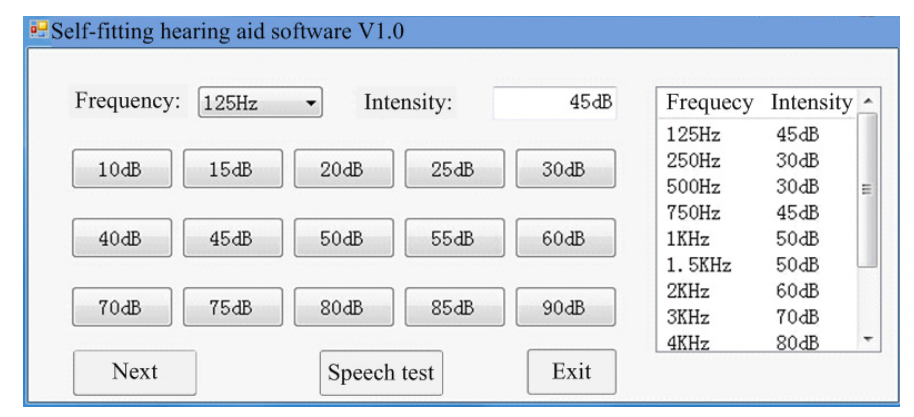

Figure 6. Interface for the pure tone test.

The speech test interface based on the proposed algorithm is shown in Figure 7. The 'Settings' button is pressed to set the algorithm parameters, which include the test speech path, crossover probability and mutation probability, etc. After the setting is finished and the 'First Group' button is pressed, the speech generated based on the first group of parameters is played, and its waveform is displayed. After the speech is played, the corresponding evaluation is performed according to the speech quality. The evaluation criteria are divided into five levels. After eight groups of speech are played in order, the 'OK' button is pressed to finish the test, and 'Next Speech' is pressed to start the new test using the new speech. The speech test is conducted until the optimal parameters are obtained. Finally, the 'Cancel' button is pressed to end the system.

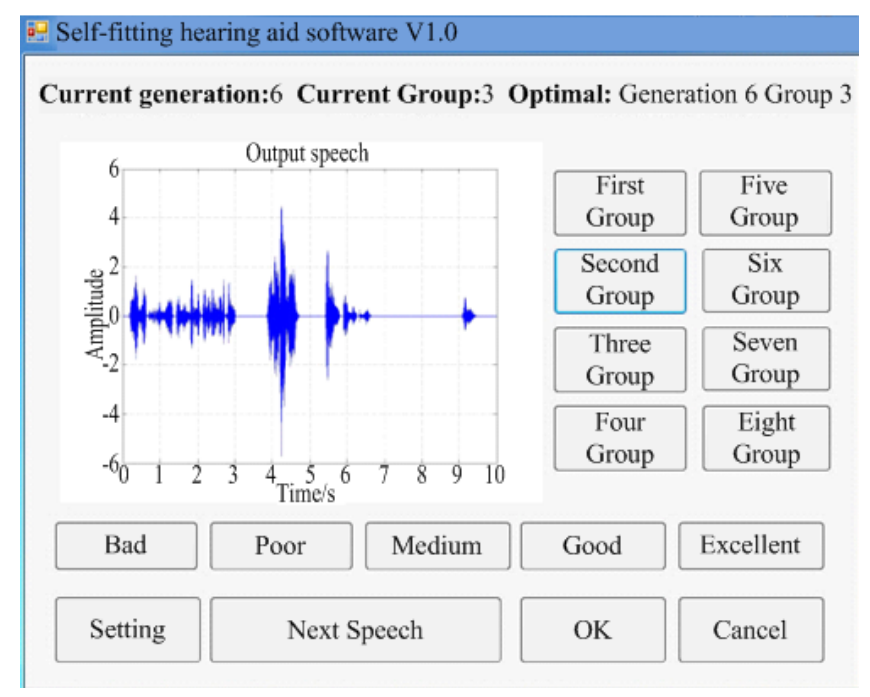

Figure 7. Speech test interface based on the proposed algorithm. 


\subsection{Speech Recognition Experiment}

\subsubsection{Procedure}

After the hearing aid is fitted, the speech recognition experiment for seven fitted subjects is done. Prior to the test, the main testing rules are explained to the subjects to understand the test purpose and method. The subjects are asked to repeat the test items and are encouraged to guess boldly if they do not hear clearly. The detailed test steps are described as follows: (1) Determine the speech recognition threshold for both of the subject's ears, or calculate the average hearing threshold of the two ears at frequencies of $0.5,1,2$, and $4 \mathrm{kHz}$; (2) Play 1 or 2 exercise tables at random, and adjust the attenuator to the subject's comfortable strength to familiarize the subject with the test requirements; (3) After playing one test item, the tester should listen to the subject's verbal response, compare with the text, and record the answer correctness. The silent interval between two test items is $4 \mathrm{~s}$, thus the subject's answer and the tester's judgment and recording should be completed in $4 \mathrm{~s}$; (4) The subject is asked to answer every test item and should give the answer of "do not hear clearly" or "do not hear" if he or she did not hear clearly or did not hear, respectively; (5) After one test sheet is finished, the speech recognition of the subject is calculated and filled in the record sheet.

Twenty-six groups of data are tested. Here, two groups of data are trained data, and others are tested data, which are divided into four groups according to four algorithms. For each algorithm, everybody has the same test data (six groups). For each person, a different algorithm has different data.

\subsubsection{Results}

Figure 8 shows the recognition rate for speech. From the figure, the recognition rate of the proposed algorithm is best, with an average value of $77 \%$. The recognition rate of patient $\mathrm{S} 7$ is the highest, reaching $86.4 \%$, and that of $\mathrm{S} 1$ is the lowest, with a value $67.0 \%$. Compared with the baseline algorithm, the recognition rates of three algorithms increase $16.2 \%, 3.4 \%$ and $4.5 \%$, respectively. In addition, the performances of the IEC algorithm for patient S1 and the GP algorithm for patient S4 are lower than that of the baseline algorithm. As seen in the figure, the performance of the GP algorithm for patient S2, S3 and S4 is lower than that of the IEC algorithm.

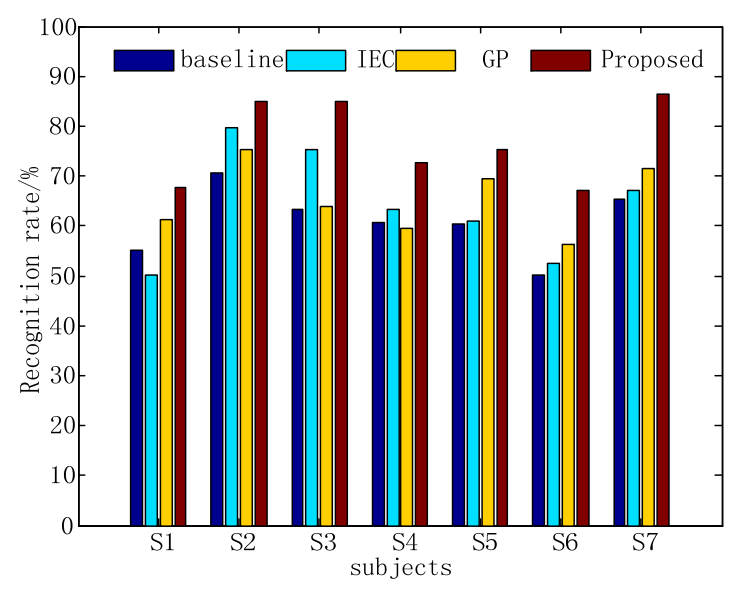

Figure 8. Comparisons of the recognition rate for speech.

To show the effect of the self-fitting algorithm comparison, two-factor variance analysis is performed, and the results are shown in Table 2. From the table, compared with the IEC algorithm, the proposed algorithm shows statistical significance, i.e., the improvement effect of the algorithm is obvious. But patient $\mathrm{S} 4$ shows no statistical significance. Relatively speaking, compared with the IEC algorithm, the improvement effect of the GP algorithm is not obvious. Only the effects of patient S4 and $\mathrm{S} 6$ are obvious, but the effect of S4 with the GP algorithm is lower than that with the IEC algorithm. 
Table 2. Statistical analysis of the recognition rate for speech.

\begin{tabular}{cccccccc}
\hline \multirow{2}{*}{ Method Comparisons } & \multicolumn{7}{c}{ Subjects ID } \\
\cline { 2 - 8 } & S1 & S2 & S3 & S4 & S5 & S6 & S7 \\
\hline I vs. G & $\Delta$ & $\Delta$ & $\Delta$ & $* * *$ & $\Delta$ & $* * *$ & $\Delta$ \\
I vs. P & $*$ & $* * *$ & $*$ & $\Delta$ & $* * *$ & $* * *$ & $* * *$ \\
G vs. P & $\Delta$ & $*$ & $\Delta$ & $* * *$ & $* * *$ & $\Delta$ & $* *$ \\
\hline
\end{tabular}

Note: Statistical significance is shown by asterisk symbols: $\Delta p>0.05$; $^{*} 0.01<p<0.05 ;{ }^{* *} 0.001<p<0.01 ;{ }^{* * *} p<0.001$.

In addition, the statistics on test time shows that the P method needs 10-20 min, which is lower than the IEC method which needs 40-60 min, and lower than the GP method which needs 20-30 min.

\subsubsection{Discussion}

Generally, the experimental results are satisfying. From the speech test, the performance of the proposed algorithm is best, which shows that the proposed optimization method is effective and stable. The reason is that the algorithm is designed based on the individual and historical data from the expert system. Relatively speaking, the other two self-fitting algorithms are not very stable. The IEC algorithm for patients S1 and the GP algorithm for patient S4 do not converge to the optimal solution. So, their performance is even worse than that of the baseline algorithm. In addition, compared with the baseline algorithm, the average recognition rates of these two algorithms are not noticeably improved, which means that these two methods have high randomness for parameters optimization.

From the two-factor variance analysis, the performance of the proposed algorithm is better than that of the other two algorithms, which also shows that the proposed algorithm is stable. However, it is obviously that stability is relative. The exceptions are S4 for the IEC algorithm and S1, S3, S6 for the GP algorithm, which means the proposed algorithm might be further improved. From stability, the GP algorithm is worst. The reason may be that the IEC algorithm uses Gaussian function parameters as the optimization parameters, which might integrate the advantages of both algorithms. However, the IEC algorithm has a slower convergent speed, which affects its performance.

Finally, compared with these two self-fitting algorithms, the fitting time of the proposed algorithm is reduced significantly. Furthermore, for the hearing aid user, the operation is more interesting because of the various audible sounds. However, the IEC algorithm has the longest time, which increases the user fatigue and affects the performance. It may be a reason for why the IEC algorithm, which integrates the advantages of both algorithms, has worst performance.

\subsection{Experiment on Environmental Sound Recognition}

\subsubsection{Results}

The experimental conditions and steps in the environmental sound assessment are nearly the same as those of speech recognition experiment. The results are shown in Figure 9. Compared with the speech test, the fitting effect of the proposed method is reduced, with an average recognition rate of $69.9 \%$, which is a $4.2 \%$ improvement compared with the GP algorithm and a $2.9 \%$ improvement compared with the IEC algorithm. However, compared with the baseline algorithm, the recognition rates of three algorithms all increase. From the figure, for the proposed algorithm, the recognition rate of patient S7 is the highest, reaching $84 \%$, and that of patient S6 is the lowest, with a value of $55.2 \%$.

For the above data, two-factor variance analysis is also conducted. The results are shown in Table 3. From the table, compared with the IEC algorithm, the proposed algorithm shows statistical significance except for patient S2. For the GP algorithm, the exceptions are S1 and S3. In addition, compared the GP algorithm with the IEC algorithm, patient S6 with the GP algorithm is decreased significantly, patient S1 and S3 are enhanced significantly, and the other patients show no significant difference. 


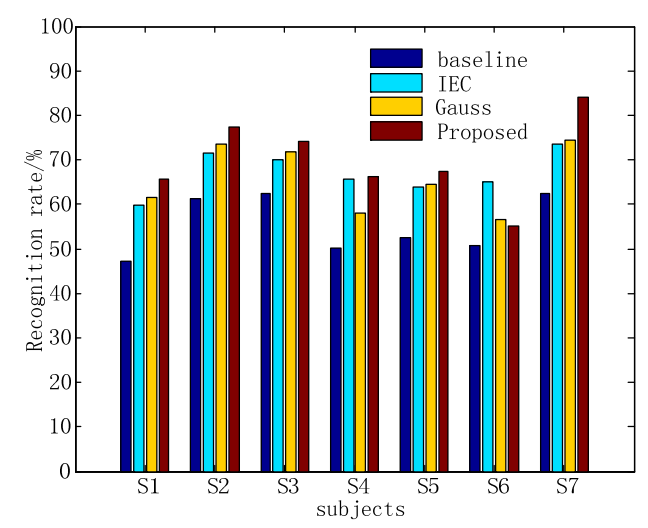

Figure 9. Recognition rate for environmental sound.

Table 3. Statistical analysis of the recognition rate for environmental sounds.

\begin{tabular}{cccccccc}
\hline \multirow{2}{*}{ Method Comparisons } & \multicolumn{8}{c}{ Subjects ID } \\
\cline { 2 - 8 } & S1 & S2 & S3 & S4 & S5 & S6 & S7 \\
\hline I vs. G & $*$ & $\Delta$ & $* *$ & $\Delta$ & $\Delta$ & $* *$ & $\Delta$ \\
I vs. P & $* * *$ & $\Delta$ & $* * *$ & $* * *$ & $*$ & $*$ & $* * *$ \\
G vs.P & $\Delta$ & $*$ & $\Delta$ & $* * *$ & $* *$ & $* * *$ & $* *$ \\
\hline
\end{tabular}

Note: Statistical significance is shown by asterisk symbols: $\Delta p>0.05 ;{ }^{*} 0.01<p<0.05 ;{ }^{* *} 0.001<p<0.01 ;{ }^{* * *} p<0.001$.

In addition, the fitting time for the environmental audio test is similar to the speech test.

\subsubsection{Discussion}

Compared with the two tests, some interesting observations are made. Firstly, on the whole, the average recognition rate of the environment test is lower than that of the speech test. The reason might be that training of the environmental sounds is not sufficient and the professional music is difficult to recognition. Secondly, the average recognition rates of the environmental sounds for patient S6 are higher than those of the speeches. After the analysis, his recognition rate for sounds is higher than that for other patients. His experience in music might show some effects. Finally, the recognition rate of the baseline algorithm decrease $5.5 \%$ for the prescription formula is designed for the speech intelligibility. However, the recognition rates for the GP algorithm and the IEC algorithm increase slightly. The reason comes from two aspects. Firstly, the average recognition rates for speeches are not very high and the self-fitting algorithm can improve the recognition rates for environmental sounds. Secondly, higher recognition rates of some patients for environmental sounds increase the average value, such as patient S6. In addition, compared with the IEC algorithm, the average recognition rate of the GP algorithm is slightly decreased, mainly due to the low recognition rates of patients S4 and S6, as shown in the figure.

\subsection{Gain Estimation}

\subsubsection{Results}

The target gains of five frequencies $(250 \mathrm{~Hz}, 500 \mathrm{~Hz}, 1 \mathrm{kHz}, 2 \mathrm{kHz}$ and $4 \mathrm{kHz})$ are compared in the experiment. Results are shown in Figure 10. From the figure, the optimal gain is computed based on the initial setting through the professional fitting software [35], and then is corrected by the audiologist for about two or three times, which will lasts several weeks. The first seven figures are the estimated gains of seven subjects, respectively, and the average gain is shown in Figure 10h. 


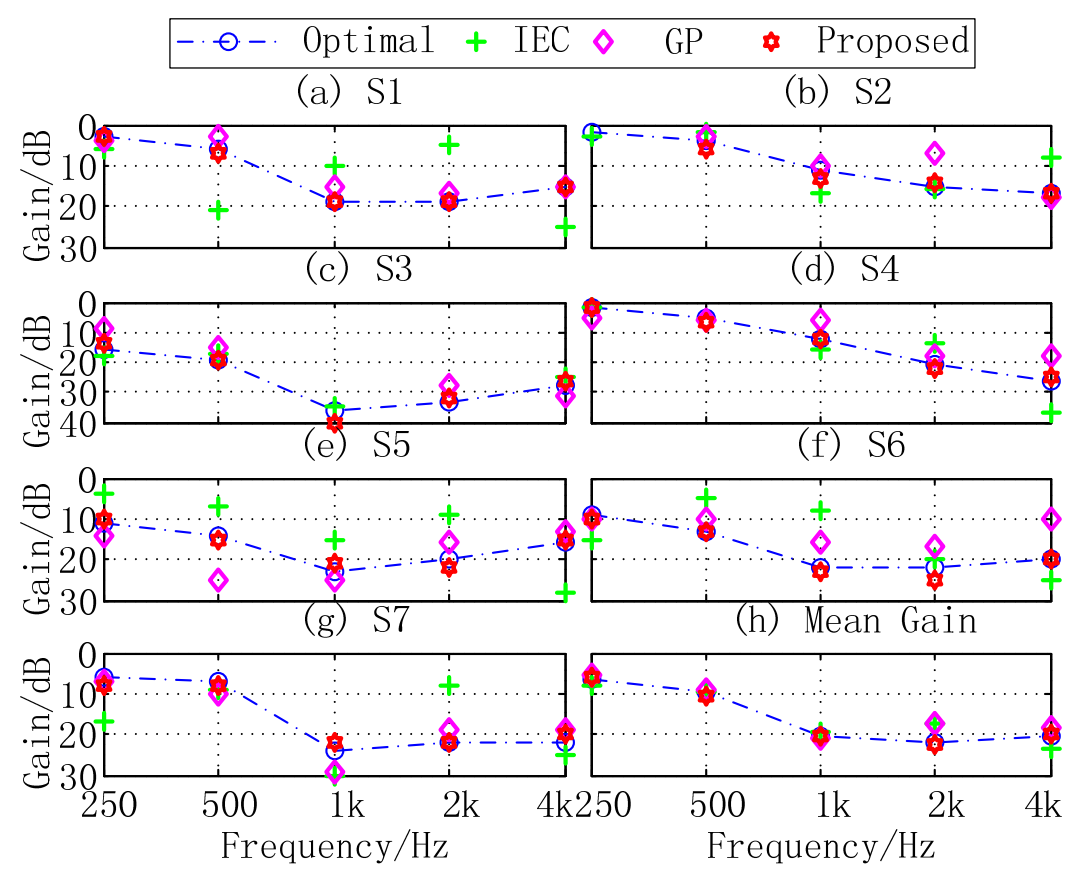

Figure 10. Gain estimation with seven subjects. (a) S1; (b) S2; (c) S3; (d) S4; (e) S5; (f) S6; (g) S7; (h) Mean Gain.

\subsubsection{Discussion}

From the results, because the proposed algorithm is designed based on the artificial intelligence and the expert system, it considers the personality attributes of patients to get the more accurate estimation. While the other two algorithms do not refer to the historical data, they have worse performance. Although the average gains with three algorithms are almost the same, the gain difference of the IEC algorithm is the largest and that of the proposed algorithm is the smallest from the single patient. The IEC algorithm and the GP algorithm have more gains fluctuation. It also can be drawn that these two algorithms are not stable. In addition, the deviations of the IEC algorithm for patients $\mathrm{S} 1, \mathrm{~S} 5$ and $\mathrm{S} 6$ are bigger, which leads to less corresponding recognition rates. Results are shown in Figures 8 and 9 .

\section{Further Discussion and Prospects for the Future}

Self-fitting hearing aid is a bold vision to improve penetration and the quality of the algorithm as well as to enhance patients' satisfaction. Self-fitting hearing aids have high values and bright application prospects, especially in developing countries where audiologists are insufficient. The design conception of self-fitting hearing aids is widely recognized by the users [36], but there are still many problems to be further studied. Potential improvements involve some aspects [37], such as accessories and algorithms of hearing aids, etc. In addition, the role of audiologists is also further discussed.

\subsection{Accessories of Hearing Aids}

The installation, debugging and usage of a self-fitting hearing aid are completely dominated by the user himself. Because the physical structures and pathological conditions of patients are different, understanding how to personalize the accessories of hearing aids is very necessary. If there are some malleable materials, it is envisaged that the mold of a hearing aid can be individually changed at any time. But there is still no related report. 
In addition, Caposecco et al. have developed some written instruction materials for the self-fitting hearing aids [17], but it is only applicative for BTE hearing aids. In the future, the most effective instructions are thought to be multi-media presentation instead of caption.

Since the debugging of self-fitting hearing aids is based on human-machine interaction, it is impractical for patients with hand disability to frequently operate the control terminal to adjust algorithm parameters for hearing aids. However, the development of brain-computer interface technology [38] may solve such problems. When the electroencephalogram (EEG) signal is amplified and translated into the external command, peripherals can be control by the mind. In this case, the fitting efficiency will be greatly improved. Although there are relatively mature brain-computer interface products, their size and power consumption are not suitable for hearing aids. There are still a lot of research works to be done to attain this goal.

\subsection{Algorithms of Hearing Aids}

Since the goal of the self-fitting algorithm is to replace the audiologists to fit hearing aids, large amounts of professional knowledge must be integrated into algorithms of hearing aids. Related algorithms consist of several aspects.

(1) Judgment of hearing loss type. Many studies have shown that there is little difference between traditional audiometer and online audiometer [39]. However, a hearing aid is not applicable for every patient, such as a patient with asymmetrical hearing loss, conductive hearing loss or mixed hearing loss. So, the patient's hearing loss type should be determined before a hearing aid is used. From the algorithm, the way to detect these hearing loss types is the major constraint for self-fitting hearing aids for the effective detection algorithm has not yet been found. Certainly, the currently safest method is that the hearing loss type is determined by an audiologist.

(2) Multi-parameter hybrid optimization strategy. With the increasing complexity of hearing aid algorithms, there are more and more parameters need to be adjusted, such as compression threshold, compression ratio, noise suppression, frequency shift, microphone mode, etc. Therefore, the joint optimization of different algorithms parameters is facing greater challenges. Although there is no similar study, parameter optimization of self-fitting hearing aids is the similar to multi-source information fusion whose decision-making is implemented with different source data. Therefore, multi-source information fusion theory can be applied to multi-parameter hybrid optimization of self-fitting hearing aids. In addition, with the development of network technology and the explosive growth of information, algorithms of distributed information fusion and multi-modal heterogeneous information fusion can be applied to parameters optimization of self-fitting hearing aids, even web server [40] and loud computing should be studied.

\subsection{Auxiliary Roles of Audiologists}

It needs to be emphasized that we do not think audiologists are completely useless in hearing aid fitting. Our viewpoint is that their functions may be not determinative in the future. The auxiliary roles of audiologists are essential, even if self-fitting hearing aids are very popular. The main roles include: (1) Understand the needs of users, examine their audition and help them build confidence, since studies show that patients' motivation is an important determinant for effective usage of hearing aids [20]; (2) After the fitting is completed, audiologists can inform the patient of some considerations, such as process strategies and methods in complex environments; (3) Provide services for future complex hearing devices, such as devices integrated with hearing aids and cochlear implantation [41].

\section{Conclusions}

To solve the problems of being time-consuming and inefficient associated with the traditional fitting algorithm, a self-fitting algorithm for digital hearing aids based on the improved IEC algorithm and expert system is proposed. The IEC algorithm can maintain the diversity of population and 
improve the prematurity problem. The expert system takes the place of the traditional audiologists to optimize the parameters of the hearing aid, which reduces user fatigue and makes the fitting process simple and effective. Moreover, to test the algorithm efficiently, the self-fitting software is designed, and a subjective speech recognition test is conducted. The results show that the fitting time is shortened, and the speech recognition rate is effectively improved.

Furthermore, the proposed algorithm can use any sounds to fit a hearing aid, which is impossible with conventional hearing aid fitting. However, the self-fitting models and algorithms need to be further studied. In our opinion, how to further reduce the fitting time and improve the fitting efficiency are still future goals. Too long fitting time and the presence of some unexpected sounds will cause user fatigue. However, it is fortunate that the algorithm efficiency can increase through different improved algorithms from some directions, such as the algorithm convergence, multi-parameter hybrid optimization strategy and expert system design, etc. Among these, multi-parameter hybrid optimization is the most important algorithm research direction. In addition, more subjects should be tested and more experiments should be repeated to get more comprehensive assessment for the proposed algorithm. It is helpful to find the problem of the algorithm to further improve its effect.

Acknowledgments: The work was supported by Six Talent Peaks Project in Jiangsu Province under Grant No. 2016-DZXX-023, the National Natural Science Foundation of China under Grant No. 61375028, 61571106 and 61673108, China Postdoctoral Science Foundation funded project under Grant No. 2016M601696, Qing Lan Project of Jiangsu Province, Jiangsu Planned Projects for Postdoctoral Research Funds under Grant No. 1601011B and Changzhou Key Technology Support Program under Grant No.CE20155041.

Author Contributions: The authors contributed equally to this work. Ruiyu Liang drafted the main manuscript and conceived the experiments. Ji Xi assisted in the design of the experiments. Li Zhao designed the experiments. Ruxue Guo and Yue Xie performed the experiments and analyzed the data.

Conflicts of Interest: The authors declare no conflict of interest.

\section{References}

1. Mcpherson, B. Innovative Technology in Hearing Instruments Matching Needs in the Developing World. Trends Amplif. 2011, 15, 209-214. [CrossRef] [PubMed]

2. Abrams, H.B. An Introduction to the Second Starkey Research Summit. Am. J. Audiol. 2012, 21, 329-330. [CrossRef]

3. Liang, R.; Xi, J.; Zhou, J.; Zou, C.; Zhao, L. An improved method to enhance high-frequency speech intelligibility in noise. Appl. Acoust. 2013, 74, 71-78. [CrossRef]

4. Rasetshwane, D.M.; Gorga, M.P.; Neely, S.T. Signal-Processing Strategy for Restoration of Cross-Channel Suppression in Hearing-Impaired Listeners. IEEE Trans. Biomed. Eng. 2014, 61, 64-75. [CrossRef] [PubMed]

5. Swanepoel, D.W.; Clark, J.L.; Koekemoer, D.; Hall, J.W., III; Krumm, M.; Ferrari, D.V.; Mcpherson, B.; Olusanya, B.O.; Mars, M.; Russo, I. Telehealth in audiology: The need and potential to reach underserved communities. Int. J. Audiol. 2010, 49, 195-202. [CrossRef] [PubMed]

6. Yoshinaga-Itano, C. Audiology in Developing Countries. Int. J. Audiol. 2012, 51, 646. [CrossRef]

7. Windmill, I.M.; Freeman, B.A. Demand for Audiology Services: 30-Yr Projections and Impact on Academic Programs. Int. J. Am. Acad. Audiol. 2013, 24, 407-416. [CrossRef] [PubMed]

8. Goulios, H.; Patuzzi, R. Audiology education and practice from an international perspective. Int. J. Audiol. 2008, 47, 647-664. [CrossRef] [PubMed]

9. Jacobs, P.G.; Saunders, G.H. New Opportunities and Challenges for Teleaudiology within Department of Veterans Affairs. J. Rehabil. Res. Dev. 2014, 51, 7-12. [CrossRef] [PubMed]

10. Singh, G.; Pichora-Fuller, M.K.; Malkowski, M.; Boretzki, M.; Launer, S. A survey of the attitudes of practitioners toward teleaudiology. Int. J. Audiol. 2014, 53, 850-860. [CrossRef] [PubMed]

11. Convery, E.; Keidser, G.; Dillon, H.; Hartley, L. A Self-Fitting Hearing Aid: Need and Concept. Trends Amplif. 2011, 15, 157-166. [CrossRef] [PubMed]

12. Keidser, G.; Convery, E. Self-Fitting Hearing Aids Status Quo and Future Predictions. Trends Hear. 2016, 20, 1-15. [CrossRef] [PubMed]

13. Eggert, A.; Gerd-Wolfgang, G.; Wolfgang, K.; Maas, R.; Wiener, P. Digital Hearing Aid and Method. U.S. Patent 447,117,1, 11 February 1984. 
14. Dreschler, W.A.; Keidser, G.; Convery, E.; Dillon, H. Client-based adjustments of hearing aid gain: The effect of different control configurations. Ear Hear. 2008, 29, 214-227. [CrossRef] [PubMed]

15. Foulad, A.; Bui, P.; Djalilian, H. Automated audiometry using Apple iOS-based application technology. Otolaryngol. Head Neck Surg. 2013, 149, 700-706. [CrossRef] [PubMed]

16. Taylor, B. Advanced user control optimizes real-world listening preferences. Hear. J. 2011, 64, $26-28$. [CrossRef]

17. Caposecco, A.; Hickson, L.; Meyer, C. Assembly and Insertion of a Self-Fitting Hearing Aid Design of Effective Instruction Materials. Trends Amplif. 2011, 15, 184-195. [CrossRef] [PubMed]

18. Takagi, H.; Ohsaki, M. Interactive evolutionary computation-based hearing aid fitting. IEEE Trans. Evolut. Comput. 2007, 11, 414-427. [CrossRef]

19. Nielsen, J.B.B.; Nielsen, J.; Larsen, J. Perception-Based Personalization of Hearing Aids Using Gaussian Processes and Active Learning. IEEE/ACM Trans. Audio Speech Lang. Process. 2015, 23, 162-173. [CrossRef]

20. Byrne, D.; Dillon, H.; Ching, T.; Katsch, R.; Keidser, G. NAL-NL1 procedure for fitting nonlinear hearing aids: Characteristics and comparisons with other procedures. Int. J. Am. Acad. Audiol. 2001, 12, 37-51.

21. Svard, I.; Spens, K.; Back, L.; Ahlner, B.; Barrenas, M. The benefit method: Fitting hearing aids in noise. Noise Health 2005, 7, 12-23. [PubMed]

22. Keidser, G.; Dillon, H.; Carter, L.; O’brien, A. NAL-NL2 Empirical Adjustments. Trends Amplif. 2012, 16, 211-223. [CrossRef] [PubMed]

23. Smeds, K.; Keidser, G.; Zakis, J.; Dillon, H.; Leijon, A.; Grant, F.; Convery, E.; Brew, C. Preferred overall loudness. II: Listening through hearing aids in field and laboratory tests. Int. J. Audiol. 2006, 45, $12-25$. [CrossRef] [PubMed]

24. Boymans, M.; Dreschler, W.A. Audiologist-driven versus patient-driven fine tuning of hearing instruments. Trends Amplif. 2012, 16, 49-58. [CrossRef] [PubMed]

25. Gan, W.-S. Applying equal-loudness compensation to the adaptive active noise control. Appl. Acoust. 2000, 61, 183-187. [CrossRef]

26. Kates, J.M. Digital Hearing Aids; Plural Publishing: San Diego, CA, USA, 2008.

27. Kowaliw, T.; Dorin, A.; Mccormack, J. Promoting creative design in interactive evolutionary computation. IEEE Trans. Evolut. Comput. 2012, 16, 523-536. [CrossRef]

28. Huang, Y.-Q.; Zhang, X.-D. Review on interactive evolutionary computation. Control Decis. 2010, 25, 1281-1286.

29. Lu, H.; Chen, L.; Song, Y.; Wu, Z.; Zou, Y. An improved crossover operator of genetic algorithm. J. PLA Univ. Sci. Technol. 2007, 8, 250-253.

30. Guo, Y.-N.; Gong, D.-W. Extraction and utilization about knowledge in hierarchical interactive genetic algorithms. Control Decis. 2007, 22, 1329-1334.

31. Cai, L.; Li, X. Improvement on crossover operation of genetic algorithms. Syst. Eng. Electron. 2006, 28, 925-928.

32. Bench, J.; Kowal, A.; Barnford, J.M. The BKB (Bamford-Kowal-Bench) sentence lists for partially-hearing children. Br. J. Audiol. 1979, 13, 108-112. [CrossRef] [PubMed]

33. Macleod, A.; Summerfield, Q. A procedure for measuring auditory and audiovisual speech-reception thresholds for sentences in noise: Rationale, evaluation, and recommendations for use. Br. J. Audiol. 1990, 24, 29-43. [CrossRef] [PubMed]

34. Xin, X. The history and present state of speech audiometry. Chin. Sci. J. Hear. Speech Rehabil. 2005, 1, $20-24$.

35. AcoSound 3.1.4.1213. Available online: http://www.acosound.com/html/zlxz/24.html (accessed on 13 March 2016).

36. Convery, E.; Keidser, G.; Hartley, L. Perception of a Self-Fitting Hearing aid Among Urban-Dwelling Hearing-Impaired Adults in a Developed Country. Trends Amplif. 2011, 15, 175-183. [CrossRef] [PubMed]

37. Liang, R.; Zou, C.; Wang, Q. Self-Fitting Hearing Aids: State of the Art, Challenges, and Future Trends. Int. J. $u$-e-Serv. Sci. Technol. 2016, 9, 1-16. [CrossRef]

38. Márquez-Chin, C.; Popovic, M.R.; Sanin, E.; Chen, R.; Lozano, A.M. Real-time two-dimensional asynchronous control of a computer cursor with a single subdural electrode. J. Spinal Cord Med. 2012, 35, 382-391. [CrossRef] [PubMed]

39. Digiovanni, J.J.; Pratt, R.M. Verification of in situ thresholds and integrated real-ear measurements. Int. J. Am. Acad. Audiol. 2010, 21, 663-670. [CrossRef] [PubMed] 
40. Calbimonte, J.-P.; Jeung, H.Y.; Corcho, O.; Aberer, K. Enabling query technologies for the semantic sensor web. Int. J. Semant. Web Inf. Syst. 2012, 8, 43-63. [CrossRef]

41. Dillon, H.; Zakis, J.A.; Mcdermott, H.; Keidser, G.; Dreschler, W.; Convery, E. The trainable hearing aid: What will it do for clients and clinicians? Hear. J. 2006, 59, 30-36. [CrossRef] 